\title{
Physical Activity, Weight Status, Diabetes and Dementia: A 34-Year Follow-Up of the Population Study of Women in Gothenburg
}

\author{
Kirsten Mehliga ${ }^{\mathrm{a}}$ Ingmar Skoog ${ }^{\mathrm{b}}$ Margda Waern $^{\mathrm{b}} \quad$ Junmei Miao Jonasson $^{\mathrm{a}}$ \\ Leif Lapidus $^{c}$ Cecilia Björkelund ${ }^{\mathrm{a}}$ Svante Östling ${ }^{\mathrm{b}}$ Lauren Lissner $^{\mathrm{a}}$ \\ a Department of Public Health and Community Medicine and ${ }^{b}$ Neuropsychiatric Epidemiology Unit, Institute for \\ Neuroscience and Physiology, Sahlgrenska Academy, University of Gothenburg, 'Department of Medicine, Sahlgrenska \\ University Hospital, Gothenburg, Sweden
}

\section{Key Words}

Dementia $\cdot$ Diabetes $\cdot$ Leisure time physical activity .

Obesity · Midlife risk factors · Population cohort

\begin{abstract}
Background: There is evidence of a synergistic interaction between obesity and sedentary lifestyle with respect to diabetes. Although diabetes is a known risk factor for dementia, it is unclear if both diseases have common aetiologies. Methods: A community-based sample of 1,448 Swedish women, aged 38-60 years and free of diabetes and dementia in 1968, was followed by means of up to 5 examinations spread over 34 years. 9.6\% of all women developed diabetes and $11.4 \%$ developed dementia (over 40,000 person-years of follow-up for each disease). Cox proportional hazard regression was used to assess the influence of selected risk factors on both diseases, and the relation between diabetes and dementia. Results: Comparing risk factors for incident diabetes and dementia, both diseases showed a synergistic association with obesity combined with a low level of leisure time physical activity [hazard ratio (HR) for interaction $=2.7,95 \%$ confidence interval $(\mathrm{Cl})=1.2-6.3$ for diabetes and $\mathrm{HR}=3.3,95 \%$ $\mathrm{Cl}=1.1-9.9$ for dementia]. Development of diabetes doubled the risk for subsequent dementia $(\mathrm{HR}=2.2,95 \% \mathrm{Cl}=$ 1.1-4.4), which was slightly reduced upon adjustment for
\end{abstract}

common risk factors. Conclusions: Shared risk factors suggest a similar aetiology for diabetes and dementia and partially explain the association between diseases.

(c) 2014 S. Karger AG, Basel

\section{Introduction}

It has been conjectured that the rising incidence of type 2 diabetes mellitus (henceforth called diabetes) and dementia are not independent evolutions but are linked to each other [1-3] and to common risk factors, including excess body weight and reduced physical activity [4]. Obesity is associated with insulin resistance, a condition which is seen as a basic process leading to vascular and metabolic disorders such as diabetes. It is known that increased physical activity can improve insulin sensitivity independently of weight [5] or weight loss [6]. It can therefore be expected that epidemiological studies might observe interactions between total body weight or fatness and physical activity on the incidence of diabetes; however, results to date are inconclusive [7]. Prospective studies have consistently shown that diabetes at baseline about doubles the risk for later dementia [3], but it is not well understood if these diseases have a similar aetiology. Evidence that they share common risk factors such as

\begin{tabular}{ll}
\hline KARGER & $\begin{array}{l}\text { ( ) 2014 S. Karger AG, Basel } \\
0251-5350 / 14 / 0424-0252 \$ 39.50 / 0 \quad \text { Karger }\end{array}$ \\
$\begin{array}{l}\text { E-Mail karger@karger.com } \\
\text { www.karger.com/ned }\end{array}$ & $\begin{array}{l}\text { This is an Open Access article licensed under the terms of the } \\
\text { Creative Commons Attribution-NonCommercial 3.0 Un- } \\
\text { ported license (CC BY-NC) (www.karger.com/OA-license), } \\
\text { applicable to the online version of the article only. Distribu- } \\
\text { tion permitted for non-commercial purposes only. }\end{array}$
\end{tabular}

Kirsten Mehlig

Department of Public Health and Community Medicine

Sahlgrenska Academy, University of Gothenburg

Box 454, SE-405 30 Gothenburg (Sweden)

E-Mail kirsten.mehlig@gu.se 
obesity and physical inactivity has been inconsistent, possibly due to the focus on individual risk factors without consideration of their interactions $[2,4,8-15]$.

This study presents results from a community-based sample of 1,448 middle-aged women, in which the incidence of diabetes and of dementia was ascertained over 34 years. The aim was to compare the midlife risk factors obesity and physical inactivity including their interaction with respect to both end points, and to confirm the relationship between diabetes and subsequent dementia.

\section{Methods}

\section{Study Design}

In 1968/1969, a representative sample of 1,622 women aged 38, $46,50,54$ or 60 years and living in Gothenburg, Sweden, was invited to the Prospective Population Study of Women in Gothenburg [16]. A total of 1,462 women (90\%) accepted the invitation and attended a comprehensive physical examination, and were also asked to complete questionnaires regarding lifestyle and medical history. Follow-up examinations were carried out in 1974/1975 $(\mathrm{n}=1,302,91 \%), 1980 / 1981(\mathrm{n}=1,156,83 \%), 1992 / 1993(\mathrm{n}=840$, $70 \%)$ and $2000 / 2001(n=665,71 \%)$, with numbers of participants and participation rates among those still alive given in parentheses $[17,18]$. In 2000/2001, women who declined examination at the clinic were offered a home visit with a reduced examination protocol, which was accepted by 167 women $(18 \%$ of those still alive [18]). Participants provided informed oral consent in 1968, 1974 and 1980, and written consent in 1992 and later. Every study wave was approved by the Regional Ethics Review Board in Gothenburg in accordance with the Declaration of Helsinki.

\section{Diagnosis of Diabetes}

At baseline and at every follow-up examination, the diabetes status was assessed and the incidence of diabetes was recorded until December 31, 2002. A subject was defined as having diabetes if the diagnosis had been made by a physician, if she was on antidiabetic medication or if two fasting blood samples showed plasma glucose concentrations $\geq 7.0 \mathrm{mmol} / \mathrm{l}$, according to the current World Health Organization definition of diabetes [19]. The information from examinations was checked for consistency with information from the Swedish Patient Register. Survivors who did not attend one of the follow-up examinations were asked to complete a short postal questionnaire including a question concerning diabetes status [20].

\section{Diagnosis of Dementia}

Psychiatric examinations were performed at every examination and included a comprehensive psychiatric interview, observations of mental symptoms and an extensive battery of neuropsychiatric tests. Close informant interviews performed via telephone in 1992/1993 and 2000/2001 included questions about changes in behaviour and intellectual function, psychiatric symptoms, activities of daily living and, in cases of dementia, age of onset and disease course. The diagnosis of dementia was based on the combined information from the psychiatric examinations and the close infor- mant interviews according to the Diagnostic and Statistical Manual of Mental Disorders (DSM-III-R) [21] and on diagnostic data from the hospital discharge register, medical records from inpatient and outpatient departments, and general practitioners' offices in Gothenburg for all women including those lost to followup [22]. Dementia diagnoses until December 31, 2002, were included in this report.

\section{Definition of Risk Factors}

Obesity was defined as body mass index (weight/height $\left.{ }^{2}, \mathrm{BMI}\right)$ $\geq 30 \mathrm{~kg} / \mathrm{m}^{2}$. Leisure time physical activity (LTPA) was assessed in 4 categories, ranging from 'almost inactive' (reading, watching TV, etc.), 'some physical activity at least $4 \mathrm{~h} /$ week' (cycling, walking, etc.) and 'regular exercise' (tennis, running, heavy gardening, etc.) to 'regular training and competitive sports' [20,23]. The questions about LTPA were identical at all examinations. Physical inactivity was defined as low LTPA, i.e. less than $4 \mathrm{~h}$ of LTPA per week versus more.

Apart from age, the following covariates were included as potential confounding factors. Higher education was defined as more than compulsory (yes vs. no) as most women at that time only completed compulsory education [24]. Smoking was assessed as present or recent smoking versus never smoking or giving up smoking more than 10 years ago. Alcohol consumption was dichotomized into current versus never or former use. Parental history of diabetes was based on self-reported information collected at all examinations (at least 1 parent with diabetes versus none). Levels of serum triglycerides are given in units of millimoles per liter. Hypertension was defined as systolic or diastolic blood pressure greater or equal to 140 or $90 \mathrm{~mm} \mathrm{Hg}$, respectively, in the sitting position after $5 \mathrm{~min}$ rest, or antihypertensive treatment. Information about diagnosis of stroke was obtained from self-reported diagnosis, medical examination and the Swedish Patient Register.

\section{Statistical Analysis}

Age-adjusted logistic or linear regression was used to examine differences in binary or continuous variables by incident diabetes and dementia status. The log transformation was applied to variables with a right-skewed distribution such as serum triglycerides. To compare risk factors for diabetes and dementia, we performed Cox proportional hazard regression of survival from baseline until diagnosis of diabetes and dementia, respectively, on the following baseline covariates: age, obesity, low LTPA, the product of obesity and low LTPA, education, smoking status, consumption of alcohol, levels of serum triglycerides, hypertension and parental history of diabetes (diabetes end point only). Due to long latency periods, we recorded the year of diagnosis rather than a specific date and used the method by Efron for tied observations. We used the SAS cumulative incidence function (CIF) macro [25] to calculate a non-parametric estimate of the CIF for dementia and for diabetes, treating death as a competing event and survival until end of follow-up as censored event. Similarly, estimates of the CIF for mortality were obtained treating diabetes or dementia as competing events. To assess diabetes as a risk factor for dementia, we compared the survival from diabetes diagnosis until dementia or censoring with survival from baseline in those without diagnosis of diabetes. Covariate values were determined at baseline (for subjects without diabetes) or at the examination closest before or at the diagnosis of diabetes. In a sensitivity analysis, we also adjusted for stroke status at the start of observation. For risk factors of pri- 
Fig. 1. Diagram for investigation of risk factors for diabetes and dementia using data from the Population Study of Women in Gothenburg. Full data on participation and loss to follow-up at all examinations is published in Bjorkelund et al. [17] and Lissner et al. [18].

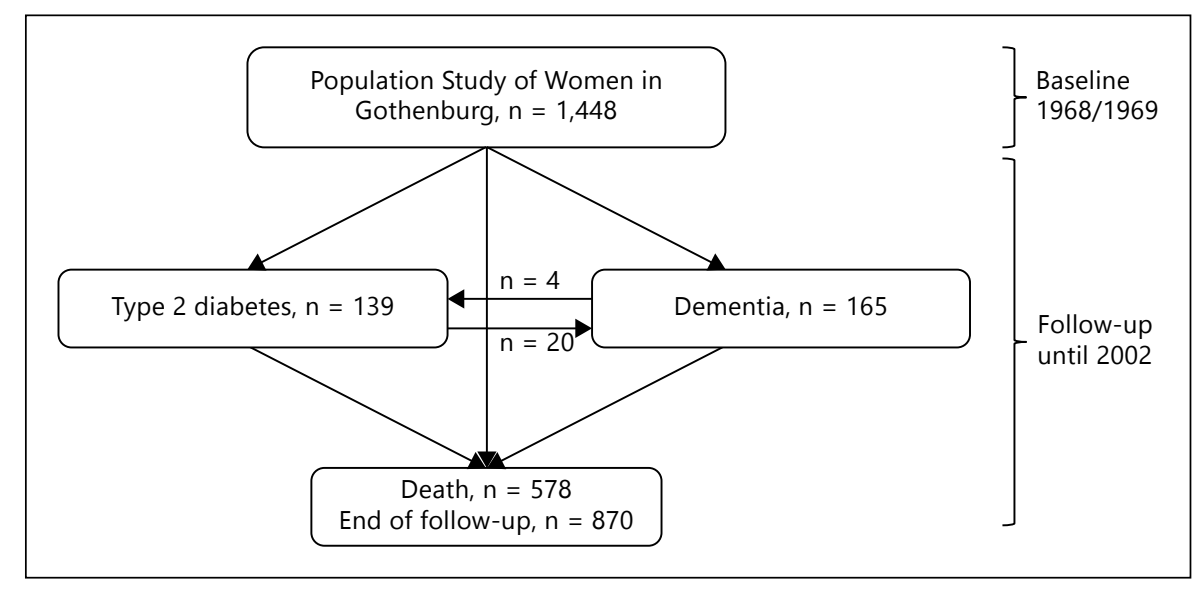

mary interest and interaction terms, the hazard ratio (HR) for dementia or diabetes is given together with the $95 \%$ confidence interval (CI). To further quantify the synergy of obesity and low LTPA with respect to diabetes and dementia, we calculated the relative excess risk due to interaction as a measure for additive interaction, with CIs as given by Zou [26], and the attributable risk due to an exposure with relative risk given by the $\mathrm{HR}$ and a prevalence $\mathrm{p}, \mathrm{AR}=\mathrm{p}(\mathrm{HR}-1) /(1+\mathrm{p}(\mathrm{HR}-1))$. All analyses were performed using SAS (version 9.3; SAS Institute, Cary, N.C., USA) and MATLAB (R2011a; MathWorks Inc.). Results with p values less than 0.05 were considered to be statistically significant (2-sided tests).

\section{Results}

Fourteen out of 153 cases of diabetes were known at baseline and excluded from the analysis. Since the youngest age at diagnosis was 43 years, incident diagnoses can be considered to be type 2 diabetes. The final analytic sample consisted of 1,448 women free of diabetes and dementia at baseline, of whom 139 developed diabetes (41,900 person-years of follow-up) and 165 developed dementia $(42,400$ person-years of follow-up; fig. 1). Twenty-four women developed both diseases, of whom 20 were diagnosed with diabetes between 0 and 20 years before the diagnosis of dementia, and the remaining up to 9 years after the diagnosis of dementia. The average age at dementia diagnosis was 76 years with a median at 77 years. The distribution of age at diagnosis was skewed to the left, with 6 diagnoses between the ages of 48 and 60 .

Table 1 gives the characteristics of subjects by status of diabetes or dementia at the end of follow-up. Incident diabetes was associated with a higher prevalence of obesity, low LTPA or their combination, higher levels of triglycerides, hypertension, parental history of diabetes, in- cident stroke and incident dementia. For incident dementia there was an indication of higher levels of obesity and low LTPA among cases, but only age, consumption of alcohol, incident stroke and incident diabetes showed a difference by dementia status.

We applied Cox proportional hazard regression to calculate the cause-specific hazard of diabetes and dementia, respectively, including death as a censored event. Compared to physically active, non-obese women, the hazard of diabetes was higher in subjects with obesity or low LTPA and highest in those with both risk factors. This is quantified in terms of a statistical interaction between obesity and low LTPA, with respect to diabetes (table 2). For dementia, there was a similar interaction between obesity and low LTPA leading to a threefold risk in women with both risk factors compared to non-obese and physically active women, in spite of the fact that there were no independent associations of either risk factor with dementia. Synergies between obesity and low LTPA were also observed based on risk differences between risk factor categories (relative excess risk due to interaction, table 2), with lack of synergy reflected by zero. The magnitude of the relative excess risk due to interaction was larger for diabetes than for dementia. The population-attributable risk of diabetes varied between 10\% for low LTPA, 12\% for obesity and $15 \%$ for their combination. In contrast, we find that the population-attributable risk of dementia for the combination of obesity and low LTPA was given by $4 \%$, compared to the category of non-obese and physically active subjects. Other vascular risk factors such as elevated levels of triglycerides (for both diseases) or hypertension (for diabetes) could not explain the association between obesity, low LTPA and dementia (data not shown).

The findings are further illustrated in figure 2 which shows the cumulative incidence of diabetes (fig. 2a) and 
Table 1. Baseline as well as incident characteristics by diabetes or dementia status at end of follow-up, among participants of the Prospective Study of Women in Gothenburg, Sweden, 1968-2002

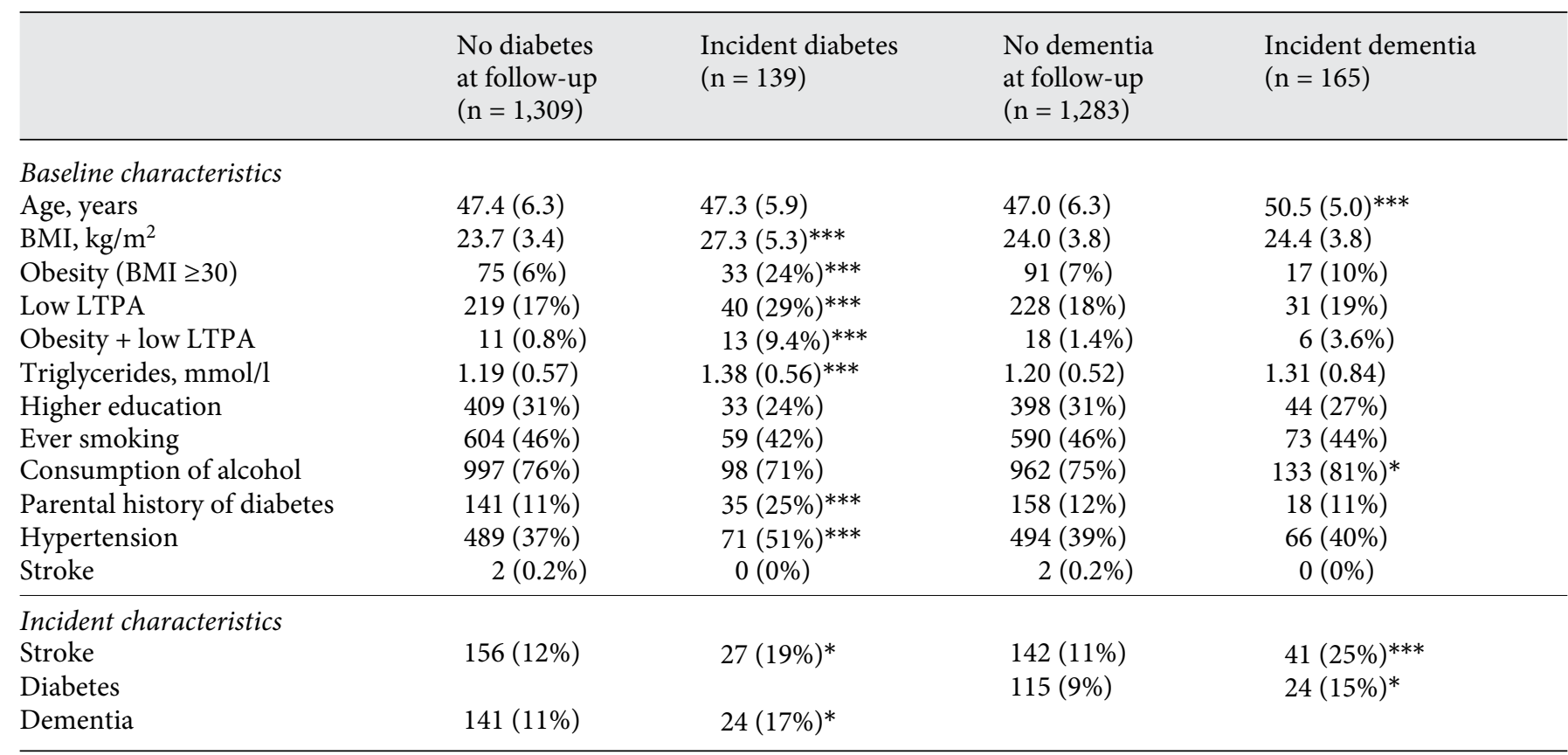

${ }^{*} \mathrm{p}<0.05,{ }^{* *} \mathrm{p}<0.01,{ }^{* * *} \mathrm{p}<0.001$ : age-adjusted $\mathrm{p}$ values comparing variables by incident endpoint.

Results are expressed as mean values with standard deviations in parentheses for continuous variables, or number of subjects with percentages in parentheses for binary variables. LTPA $=$ Leisure time physical activity.

Table 2. HR of diabetes or dementia, respectively, for the different combinations of obesity and physical inactivity (upper part) and HR of dementia following diabetes (lower part)

\begin{tabular}{|c|c|c|c|c|c|c|}
\hline Risk factor & \multicolumn{6}{|l|}{ Endpoint } \\
\hline Non-obese, active & $79(7 \%)$ & 1 (ref.) & & $123(11 \%)$ & 1 (ref.) & \\
\hline Non-obese, inactive & $27(11 \%)$ & $1.79^{1}$ & $1.15-2.79$ & $25(11 \%)$ & $1.04^{1}$ & $0.67-1.61$ \\
\hline \multirow{2}{*}{$\begin{array}{l}\text { Obese } \times \text { inactive } \\
\text { RERI }\end{array}$} & & $2.69^{1}$ & $1.15-6.30$ & & $3.26^{1}$ & $1.07-9.94$ \\
\hline & & $8.48^{1}$ & $3.16-18.3$ & & $2.29^{1}$ & $0.21-6.62$ \\
\hline \multirow[t]{2}{*}{ Diabetes } & - & - & - & $24(17 \%)$ & $2.19^{2}$ & $1.10-4.36$ \\
\hline & - & - & - & & $1.95^{3}$ & $0.94-4.05$ \\
\hline
\end{tabular}

Figures in parentheses indicate row percentages. The interaction analysis includes the estimate for the product term in the multiplicative model as well as an estimate for additive interaction (relative excess risk due to interaction, RERI, reference value for lack of interaction $=0$ ); 'inactive' = low LTPA, remainder referred to as 'active'.

${ }^{1}$ Adjusted for baseline covariates age, education, smoking, consumption of alcohol, triglycerides, hypertension and parental history of diabetes (diabetes only).

${ }^{2}$ Adjusted for age at start of observation (baseline or diagnosis of diabetes, respectively).

${ }^{3}$ Adjusted for age, education, smoking, consumption of alcohol, triglycerides, hypertension, obesity, inactivity, and obesity $\times$ inactivity, at start of observation.

Physical Activity, Obesity, Diabetes and Dementia
Neuroepidemiology 2014;42:252-259 DOI: $10.1159 / 000362201$ 


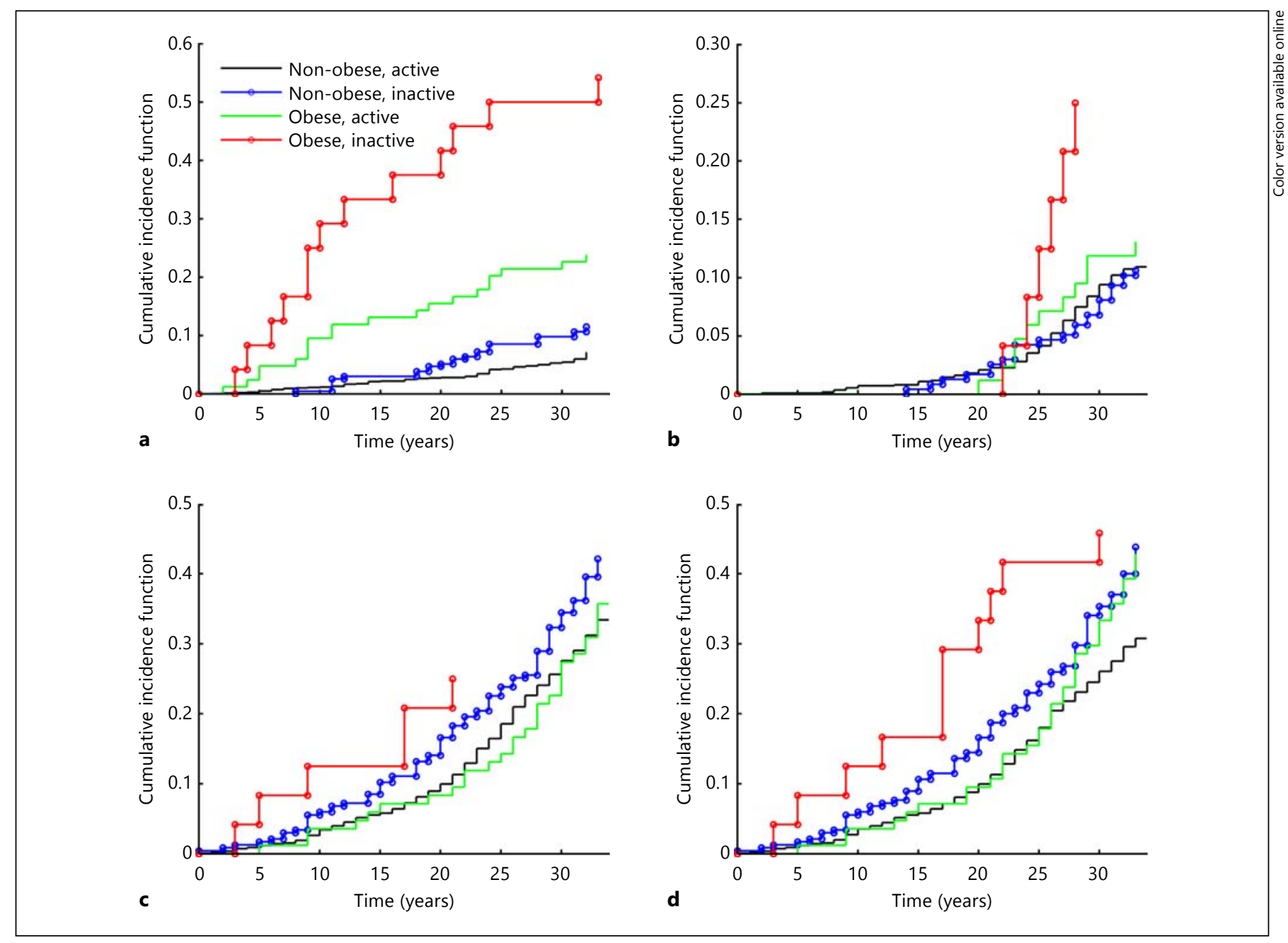

Fig. 2. CIFs for diabetes (a) and dementia (b) versus survival time from baseline, including mortality as a competing risk. c, d CIFs for mortality with diabetes and dementia considered as competing risk, respectively, i.e. diabetes-free mortality (c) and dementia-free mortality (d). 'Inactive' refers to 'low LTPA', and the remainder is referred to as 'active'.

were still alive failed to attend a scheduled follow-up examination. When those without a subsequent diagnosis were treated as censored in the year last seen, the HRs of diabetes and dementia hardly differed from the results given in table 2 (data not shown).

Finally, we examined whether diabetes is a precursor for dementia. Figure 3 shows that the age-adjusted cumulative hazard function for dementia was higher for subjects with diabetes than for those without at all points in time. A diagnosis of diabetes in midlife or later doubled the risk of dementia compared to the risk in non-diabetic women when adjusted for age at the start of observation (table 2). The HR for dementia was only slightly reduced when adjusted for confounding variables, including the shared risk factors obesity, low LTPA and their interac- 


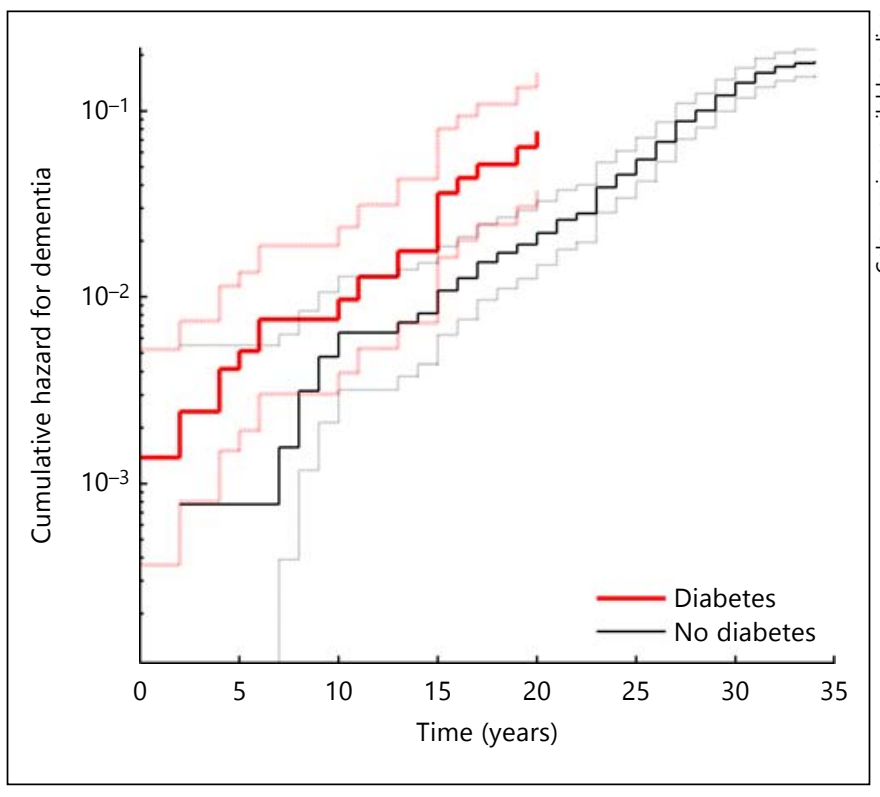

Fig. 3. Cumulative hazard function for dementia stratified by diabetes status and adjusted for age at start of observation (baseline or diagnosis of diabetes, respectively).

tion (last line, table 2), and remained unchanged after further adjustment for stroke status at the start of observation (data not shown). Conversely, the larger part (73\%) of the association between obesity and low LTPA and dementia was explained by the mediating effect of diabetes (data not shown).

\section{Discussion}

In this study, we followed a population-based cohort of 1,448 middle-aged women over 34 years identifying 139 incident cases of type 2 diabetes and 165 cases of dementia. We found similar joint risk factors for both, diabetes and dementia, namely midlife obesity in combination with low LTPA. While obesity and low LTPA were strong risk factors for diabetes individually and in combination, we found that obesity and low LTPA were risk factors for dementia only if combined with each other. Due to the late onset of disease, mortality is a stronger competing risk for dementia than it is in the case of diabetes. This may be one of the reasons why our study, with its long follow-up time, was able to demonstrate an interaction between obesity and physical activity on dementia. The synergy between obesity and low LTPA measured in terms of absolute risk difference or population-attributable risk was about 3 times larger for diabetes than for dementia. Finally, we showed that the diagnosis of diabetes doubled the risk for dementia, which is consistent with results from earlier prospective studies [3]. Common risk factors and their interaction attenuated the association between diabetes and dementia only slightly.

A perturbed insulin metabolism leading to insulin resistance both peripherally and in the brain has been proposed as an intermediate condition for both diabetes and dementia [27-30]. The observed interaction of obesity and low LTPA, both associated with e.g. enlarged abdominal adipocytes, supports the hypothesis of insulin resistance as a common pathology. The fact that obesity and low LTPA were risk factors for dementia only if observed simultaneously could indicate that additional pathologies must join to insulin resistance in order to cause dementia. For instance, the metabolic changes due to obesity, which by themselves are causing diabetes, may progress to dementia if oxidative stress due to physical inactivity is added. It is also possible that effects of individual risk factors are 'washed out' due to change of lifestyle during followup, and that only the combined effect of both risk factors can be observed after 20 years of follow-up. While the relative measure of risk due to the combination of obesity and low LTPA is of similar magnitude with regard to diabetes and dementia, absolute measures based on the risk differences or the population-attributable risk indicate a stronger association of the risk factor combination with diabetes than with dementia. Among possible explanations, e.g. loss of correlation between baseline exposure and outcome in high age in the case of dementia, or a larger number of necessary causes for dementia compared to diabetes, we emphasize that the prevalence of the exposure, i.e. obesity and low LTPA, are likely to have increased in today's societies, which will give larger estimates for the population-attributable risk of these risk factors with respect to both diseases. As an interesting observation we note that low LTPA without obesity was the second largest risk factor for mortality, with diabetes or dementia considered as competing event. It appears that the risk due to low LTPA surpasses that due to obesity for causes of death not mediated by diabetes (or dementia), such as upper respiratory tract infections [31] or certain types of cancer [32,33].

The strengths of our study include the long follow-up of a community-based cohort, with participation rates of $90 \%$ at baseline, and higher or equal to $70 \%$ at follow-up examinations, which exceed participation rates of modern cohorts [34], and accurate information on predicting variables and end points. The assessment of risk factors in midlife reduces the risk of reverse causation with respect 
to dementia, that is progressing cognitive decline leading to reduced physical activity. The small sample size is the major limitation. In addition, the assessment of physical activity by 4 categories of self-reported LTPA at baseline is rather crude and subject to reporting errors. Future studies should include more objective measures of physical activity. Finally, it must be acknowledged that risk profiles are different in contemporary cohorts. For instance, a higher average level of education can be expected for today's female population, related to a lower risk for dementia. However, local studies $[35,36]$ have shown an increase in central obesity in the general adult population, in spite of concurrent trends of more physical activity. As central obesity is both a marker for insufficient physical activity and a risk factor for diabetes, we believe that the findings based on middle-aged women from the late sixties are even more relevant for present societies.

\section{Conclusion}

In summary, the evidence is strong that obesity and physical inactivity are common risk factors for both type 2 diabetes and dementia although they can only partly explain the connection between the diseases. Nevertheless, both obesity and physical inactivity are modifiable but prevalent conditions, and our data suggest that their reduction could effectively reduce or postpone the incidence of both diseases.

\section{Acknowledgements}

The authors acknowledge financial support from the Swedish Research Council (11267, 2005-8460, 825-2007-7462), Swedish Council for Working Life and Social Research (2001-2835, 20012646, 2003-0234, 2004-0150, 2006-0020, 2008-1229, 2004-0145, 2006-0596, 2008-1111, 2010-0870; EpiLife 2006-1506; WISH 2007-1958), Swedish Brain Power, the Alzheimer's Association Zenith Award (ZEN-01-3151), the Alzheimer's Association Stephanie B. Overstreet Scholars (IIRG-00-2159), the Bank of Sweden Tercentenary Foundation, Stiftelsen Söderström-Königska Sjukhemmet, Stiftelsen för Gamla Tjänarinnor, Handlanden Hjalmar Svenssons Forskningsfond and Stiftelsen Professor Bror Gadelius' Minnesfond.

\section{Disclosure Statement}

The authors declare that they have no conflict of interest.

\section{References}

1 Biessels GJ, Staekenborg S, Brunner E, Brayne C, Scheltens P: Risk of dementia in diabetes mellitus: a systematic review. Lancet Neurol 2006;5:64-74.

2 Sahathevan R, Brodtmann A, Donnan GA: Dementia, stroke, and vascular risk factors: a review. Int J Stroke 2012;7:61-73.

$\checkmark 3$ Cheng G, Huang C, Deng H, Wang H: Diabetes as a risk factor for dementia and mild cognitive impairment: a meta-analysis of longitudinal studies. Intern Med J 2012;42: 484-491.

$\checkmark 4 \mathrm{Li} \mathrm{L}$, Holscher C: Common pathological processes in Alzheimer disease and type 2 diabetes: a review. Brain Res Rev 2007;56:384-402.

5 LaMonte MJ, Blair SN, Church TS: Physical activity and diabetes prevention. J Appl Physiol 2005;99:1205-1213.

6 Zoeller RF: Physical activity and obesity: their interaction and implications for disease risk and the role of physical activity in healthy weight management. Am J Lifestyle Med 2007;1:437-446.

7 Qin L, Knol MJ, Corpeleijn E, Stolk RP: Does physical activity modify the risk of obesity for type 2 diabetes: a review of epidemiological data. Eur J Epidemiol 2010;25:5-12.

$\checkmark 8$ Barnes DE, Yaffe K: The projected effect of risk factor reduction on Alzheimer's dis- ease prevalence. Lancet Neurol 2011;10: 819-828.

-9 Luchsinger JA, Cheng D, Tang MX, Schupf $\mathrm{N}$, Mayeux R: Central obesity in the elderly is related to late-onset Alzheimer disease. Alzheimer Dis Assoc Disord 2012;26:101105.

10 Scarmeas N, Luchsinger JA, Brickman AM, Cosentino S, Schupf N, Xin-Tang M, Gu Y, Stern Y: Physical activity and Alzheimer disease course. Am J Geriatr Psychiatry 2011;19: 471-481.

-11 Simons LA, Simons J, McCallum J, Friedlander Y: Lifestyle factors and risk of dementia: Dubbo study of the elderly. Med J Aust 2006; 184:68-70.

12 Whitmer RA, Sidney S, Selby J, Johnston SC, Yaffe K: Midlife cardiovascular risk factors and risk of dementia in late life. Neurology 2005;64:277-281.

$13 \mathrm{Xu}$ WL, Atti AR, Gatz M, Pedersen NL, Johansson B, Fratiglioni L: Midlife overweight and obesity increase late-life dementia risk: a population-based twin study. Neurology 2011;76:1568-1574.

14 Van den Berg E, Kloppenborg RP, Kessels RP, Kappelle LJ, Biessels GJ: Type 2 diabetes mellitus, hypertension, dyslipidemia and obesity: a systematic comparison of their impact on cognition. Biochim Biophys Acta 2009;1792: 470-481.

15 Gustafson D, Rothenberg E, Blennow K, Steen B, Skoog I: An 18-year follow-up of overweight and risk of Alzheimer disease. Arch Intern Med 2003;163:1524-1528.

16 Bengtsson C, Blohme G, Hallberg L, Hallstrom $\mathrm{T}$, Isaksson $\mathrm{B}$, Korsan-Bengtsen $\mathrm{K}$, Rybo G, Tibblin E, Tibblin G, Westerberg H: The study of women in Gothenburg 19681969: a population study. General design, purpose and sampling results. Acta Med Scand 1973;193:311-318.

17 Bjorkelund C, Bondyr-Carlsson D, Lapidus L, Lissner L, Mansson J, Skoog I, Bengtsson C: Sleep disturbances in midlife unrelated to 32 year diabetes incidence: the prospective population study of women in Gothenburg. Diabetes Care 2005;28:2739-2744.

18 Lissner L, Skoog I, Andersson K, Beckman N, Sundh V, Waern M, Zylberstein DE, Bengtsson C, Bjorkelund C: Participation bias in longitudinal studies: experience from the population study of women in Gothenburg, Sweden. Scand J Primary Health Care 2003;21:242-247.

19 IDF/WHO: Definition and Diagnosis of Diabetes Mellitus and Intermediate Hyperglycaemia. Report of a WHO/IDF Consultation. Geneva, WHO, 2006. 
20 Lapidus L, Bengtsson C, Bergfors E, Bjorkelund C, Spak F, Lissner L: Alcohol intake among women and its relationship to diabetes incidence and all-cause mortality: The 32year follow-up of a population study of women in Gothenburg, Sweden. Diabetes Care 2005;28:2230-2235.

21 American Psychiatric Association: Diagnositic and Statistical Manual of Mental Disorders, ed 3, revised (DSM-III-R). Washington, American Psychiatric Association, 1987.

22 Skoog I, Nilsson L, Palmertz B, Andreasson LA, Svanborg A: A population-based study of dementia in 85-year-olds. N Engl J Med 1993; 328:153-158.

23 Lissner L, Potischman N, Troiano R, Bengtsson C: Recall of physical activity in the distant past: the 32-year follow-up of the prospective population study of women in Göteborg, Sweden. Am J Epidemiol 2004;159:304-307.

24 Cabrera C, Helgesson O, Wedel H, Bjorkelund C, Bengtsson C, Lissner L: Socioeconomic status and mortality in Swedish women: opposing trends for cardiovascular disease and cancer. Epidemiology 2001;12: 532-536.
25 Lin G, So Y, Johnston G: Analyzing survival data with competing risks using SAS software. Paper 344-2012. Orlando, SAS Global Forum 2012.

26 Zou GY: On the estimation of additive interaction by use of the four-by-two table and beyond. Am J Epidemiol 2008;168:212-224.

27 De la Monte SM: Contributions of brain insulin resistance and deficiency in amyloid-related neurodegeneration in Alzheimer's disease. Drugs 2012;72:49-66.

28 Luchsinger JA: Insulin resistance, type 2 diabetes, and $\mathrm{AD}$ : cerebrovascular disease or neurodegeneration? Neurology 2010;75:758759.

29 Manolopoulos KN, Klotz LO, Korsten P, Bornstein SR, Barthel A: Linking Alzheimer's disease to insulin resistance: the FoxO response to oxidative stress. Mol Psychiatry 2010;15:1046-1052.

30 Sato N, Takeda S, Uchio-Yamada K, Ueda H, Fujisawa T, Rakugi H, Morishita R: Role of insulin signaling in the interaction between Alzheimer disease and diabetes mellitus: a missing link to therapeutic potential. Curr Aging Sci 2011;4:118-127.

31 Nieman DC, Pedersen BK: Exercise and immune function. Recent developments. Sports Med 1999;27:73-80.
32 Lee IM, Shiroma EJ, Lobelo F, Puska P, Blair SN, Katzmarzyk PT: Effect of physical inactivity on major non-communicable diseases worldwide: an analysis of burden of disease and life expectancy. Lancet 2012;380:219229.

33 Furberg AS, Thune I: Metabolic abnormalities (hypertension, hyperglycemia and overweight), lifestyle (high energy intake and physical inactivity) and endometrial cancer risk in a Norwegian cohort. Int J Cancer 2003; 104:669-676.

34 Larsen SB, Dalton SO, Schuz J, Christensen J, Overvad K, Tjonneland A, Johansen C, Olsen A: Mortality among participants and nonparticipants in a prospective cohort study. Eur J Epidemiol 2012;27:837-845.

35 Berg C, Rosengren A, Aires N, Lappas G, Toren $\mathrm{K}$, Thelle $\mathrm{D}$, Lissner L: Trends in overweight and obesity from 1985 to 2002 in Göteborg, West Sweden. Int J Obes 2005;29:916924.

36 Lissner L, Sjoberg A, Schutze M, Lapidus L, Hulthen L, Bjorkelund C: Diet, obesity and obesogenic trends in two generations of Swedish women. Eur J Nutr 2008;47:424-431. 